\title{
ON HYPERBOLICALLY KAEHLERIAN BI-RECURRENT AND BI-SYMMETRIC SPACES
}

\author{
K.S.Rawat ${ }^{1}$ Mukesh Kumar $^{2}$ and Nitin Uniyal ${ }^{3}$ \\ Department of Mathematics \\ H.N.B.Garhwal University Campus \\ Badshahi Thaul, Tehri (Garhwal) -249199 \\ Uttarakhand, India.
}

\begin{abstract}
Tachibana (1967) have studied on the Bochner curvature tensor. Singh (1971-72) studied on Kaehlerian recurrent and Ricci-recurrent spaces of second order. Further, Negi and Rawat (1994) have been studied some bi-recurrent and bi-sym metric properties in a Kaehlerian space.

In the present paper, we have been studied Hyperbolically Kaehlerian bi-recurrent and bi-symmetric spaces also several theorems have been established and proved therein.
\end{abstract}

Keywords: Bi-recurrent, bi-symmetric, Hyperbolically Kaehlerian Space, Kaehlerian space, Sasakian space.

\section{Introduction}

Spaces with additional structures which arise in theoretical Physics play an important part in the theory of Riemannian spaces $V_{n}$. Such spaces are, in particular, "Classical" Kaehlerian and Sasakian spaces as well as hyperbolically Kaehlerian and Hyperbolically Sasakian spaces.

Definition (1.1): A four- dimensional Riemannian space $K_{n}$ is called a hyperbolically Kaehlerian space if, along with Riemannian metric tensor $g_{i j}$, a complex structure tensor $F_{i}^{\text {h }}$ satisfies the following conditions:

$$
\begin{gathered}
F_{k}^{h} F_{i}^{k}=\delta_{i}^{h}, \\
g_{k i} F_{j}^{k}+g_{k j} F_{i}^{k}=0, \\
F_{i, j}^{h}=0,
\end{gathered}
$$

where the comma (, ) followed by an index denotes the operator of covariant differentiation w.r.to the Riemannian metric tensor.

Definition (1.2): An odd-dimensional Riemannian space $S_{n \text { n }}$ is called a hyperbolically Sasakian space if, along with metric tensor $g_{i j}$, a complex structure tensor $F_{i}^{h}$ satisfies the following conditions :

$$
\begin{aligned}
& F_{k}^{h} F_{i}^{k}=\delta_{i}^{h}-X^{h} X_{i}, \\
& F_{k}^{h} X^{k}=0, \\
& X^{k} X_{k}=1, \\
& g_{k i} F_{j}^{k}+g_{k j} F_{i j}^{k}=0, \\
& F_{i j}^{h}=X^{h} g_{i j}-\delta_{j}^{h} X_{i},
\end{aligned}
$$

where $X_{\mathrm{i}} \equiv X^{k} g_{k i}$ is some vector.

Differentiating (1.4), it is easy to establish that $F_{i}^{h}=X_{i j}^{k}$. This definition of Sasakian spaces is over determined.

The Riemannian curvature tensor field $R_{\mathrm{fj} k}^{\mathrm{h}}$ is defined as

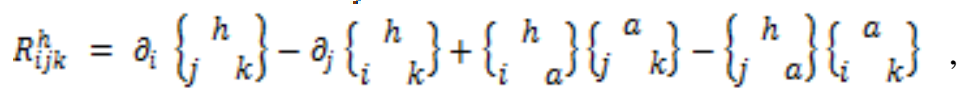

where $\partial_{\mathrm{i}}=\frac{\partial}{\partial x^{i}}$ and $\left\{x^{i}\right\}$ denotes the real local coordinates.

The Ricci tensor and the Scalar curvature are respectively given by

$$
R_{\mathrm{i} j}=R_{\mathrm{aij} j}^{\mathrm{a}} \text { and } \mathrm{R}=g^{i j} R_{\mathrm{ij}} \text {. }
$$

If we define a tensor $S_{i j}$ by

$S_{i j}=F_{i}^{a} R_{a j}$,

Then we have 


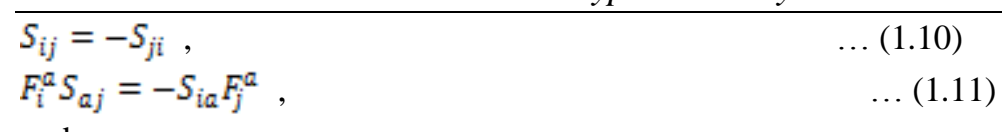

and

$F_{\mathrm{i}}^{a} S_{j k a}=R_{j i_{k} k}-R_{\mathrm{ki}_{j} j}$.

It has been verified in Yano([5]) pages 63,68 that the metric tensor $g_{\mathrm{i} j}$ and the Ricci-tensor denoted by $R_{i j}$ are hybrid in $i$ and $j$. Therefore, we get

$g_{\mathrm{ij}}=g_{\mathrm{rg}} F_{\mathrm{i}}^{\gamma} F_{j}^{g}$,

and

$$
R_{\mathrm{ij}}=R_{r g} F_{i}^{\gamma} F_{j}^{g} \text {, }
$$

The Holomorphically Projective curvature tensor $P_{\mathrm{i} f k}^{\mathrm{h}}$ is given by

$P_{\mathrm{i} j k}^{h}=R_{i j k}^{h}+\frac{1}{(n+2)}\left(R_{i k} \delta_{j}^{h}-R_{j k} \delta_{i}^{h}+S_{i k} F_{j}^{h}-S_{j k} F_{i}^{h}+2 S_{i j} F_{k}^{h}\right)$,

The Tachibana H-Concircular curvature tensor and the Weyl-Conformal curvature tensors are respectively given by

$$
\begin{aligned}
& T_{i j k}^{h}=R_{i j k}^{h}+\frac{R}{n(n+2)}\left(g_{i k} \delta_{j}^{h}-g_{j k} \delta_{i}^{h}+F_{i k} F_{j}^{h}-F_{j k} F_{i}^{h}+2 F_{i j} F_{k}^{h}\right), \\
& \text { and } \\
& C_{i j k}^{h}=\frac{1}{(n-)}\left(R_{i k} \delta_{j}^{h}-R_{j k} \delta_{i}^{h}+g_{i k} R_{j}^{h}-g_{j k} R_{i}^{h}\right)-\frac{n}{(n-1)(n-2)}\left(g_{i k} \delta_{j}^{h}-g_{j k} \delta_{i j}^{h}\right),
\end{aligned}
$$

There is a Weyl-Concircular curvature tensor given by (Sinha, 1971)

$Z_{i j k}^{h}=R_{i j k}^{i}+\frac{R}{n(n-1)}\left(g_{i k} \delta_{j}^{h}-g_{j k} \delta_{i}^{h}\right)$.

If, we put

$L_{\mathrm{i} j}=R_{\mathrm{i} j}-\frac{R}{\mathrm{n}} g_{\mathrm{i} j}$

and

$M_{i j}=F_{i}^{\alpha} L_{a j j}=S_{i j}-\frac{R}{n} F_{i}$

Then from (1.15), (1.16), (1.16), (1.19) and (1.20), we get

$P_{i j k}^{h}=T_{i j k}^{h}+\frac{1}{(n+2)}\left(L_{i k} \delta_{j}^{h}-L_{j k} \delta_{i}^{h}+M_{i k} F_{j}^{h}-M_{j k} F_{i j}^{h}+2 M_{i j} F_{k}^{h}\right)$.

and with the help of (1.17), (1.18), (1.19) and (1.20), we have

$C_{i j k}^{h}=Z_{i j k}^{h}+\frac{1}{(n-2)}\left(L_{i k} \delta_{j}^{h}-L_{j k} \delta_{i}^{h}+g_{i k} L_{j}^{h}-g_{j k} L_{i}^{h}\right)$.

Now, we shall use the following:

Definition (1.3). A hyperbolically Kaehlerian space $K_{\mathrm{n}}$ is said to be bi-recurrent, if we have $R_{i j k_{\alpha} a b}^{h}-\lambda_{a b} R_{i j k}^{h}=0$, or, equivalently $R_{i j k l_{j} a b}-\lambda_{a b} R_{i j k l}=0$.

for some non-zero tensor field $\lambda_{a b}$, and is known as recurrence tensor field.

A hyperbolically Kaehlerian space whose Ricci-tensor $R_{\mathrm{i} j}$ satisfies the equation

$R_{i j a b}-\lambda_{a b} R_{i j}=0$,

for some non-zero tensor $\lambda_{a b}$, is called hyperbolically Kaehlerian Ricci-bi-recurrent space. the above equation by $g^{i j}$, we have

$R_{a b}-\lambda_{a b} R=0$.

\section{Hyperbolically Kaehlerian Spaces With Bi-Recurrent Properties}

Definition (2.1). A hyperbolically Kaehlerian space satisfying the relation

$$
P_{i j k, a b}^{h}-\lambda_{a b} P_{i j k}^{h}=0 \text {, or, equivalently } P_{i j k l_{j} a b}-\lambda_{a b} P_{i j k l}=0 \text {, }
$$

For some non-zero tensor field $\lambda_{a b}$, will be called hyperbolically Kaehlerian projective bi-recurrent space.

Definition (2.2). A hyperbolically Kaehlerian space satisfying the relation

$$
T_{i j k, a b}^{h}-\lambda_{a b} T_{i j k}^{h}=0 \text {, or, equivalently } T_{i j k l_{d} a b}-\lambda_{a b} T_{i j k i}=0,
$$

For some non-zero tensor field $\lambda_{a b}$, will be called hyperbolically Kaehlerian space with Tachibana HConcircular bi-recurrent space.

Definition (2.3). A hyperbolically Kaehlerian space satisfying the relation

$$
C_{i j k a b}^{h}-\lambda_{a b} C_{i j k}^{h}=0 \text {, or equivalently } C_{i j k l a b}-\lambda_{a b} C_{i j k l}=0,
$$

For some non-zero tensor field $\lambda_{a b}$, will be called hyperbolically Kaehlerian space with bi-recurrent WeylConformal curvature tensor. 
Definition (2.4). A hyperbolically Kaehlerian space satisfying the relation

$Z_{i j k, a b}^{h}-\lambda_{a b} Z_{i j k}^{h}=0$, or, equivalently $Z_{i j k l a b}-\lambda_{a b} Z_{i j k l}=0$,

For some non-zero recurrence tensor field $\lambda_{a b}$, will be called hyperbolically Kaehlerian space with birecurrent Weyl-Concircular curvature tensor.

Now, we have the following:

Theorem (2.1) : If a hyperbolically Kaehlerian space satisfying any two of the following properties:

(i) the space is hyperbolically Kaehlerian Ricci-bi-recurrent ,

(ii) the space is hyperbolically Kaehlerian projective bi-recurrent,

(iii) the space is hyperbolically Kaehlerian Tachibana H-Concircular bi-recurrent ,then it must also satisfy the third.

Proof. Differentiating (1.21) covariantly w.r.to $x^{\llbracket}$, again differentiate the result thus obtained covariantly w.r.to $x^{b}$, we have

$$
P_{i j k_{a} a b}^{h}=T_{i j k_{i} a b}^{h}=\frac{1}{(n+2)}\left(L_{i k_{a} a b} \delta_{j}^{h}-L_{j k_{a} a b} \delta_{i}^{h}+M_{i k_{a} a b} F_{j}^{h}-M_{j k_{a} a b} F_{i}^{h}+2 M_{i j a b} F_{k}^{h}\right) \text {, }
$$

Multiplying (1.21) with $\lambda_{a b}$ and subtracting the result thus obtained from (2.5), we have

$$
\begin{aligned}
P_{i j k_{a} a b}^{h}-\lambda_{a b} P_{i j k}^{h}= & T_{i j k_{a} a b}^{h}-\lambda_{a b} T_{i j k}^{h}+\frac{1}{(n+2)}\left[\left(L_{i k_{a} a b}-\lambda_{a b} L_{i k}\right) \delta_{j}^{h}-\left(L_{j k, a b}-\lambda_{a b} L_{j k}\right) \delta_{i}^{h}\right. \\
& \left.+\left(M_{i k_{a} a b}-\lambda_{a b} M_{i k}\right) F_{j}^{h}-\left(M_{j k_{a} a b}-\lambda_{a b} M_{j k}\right) F_{i}^{h}+2\left(M_{i j a b}-\lambda_{a b} M_{i j}\right) F_{k}^{h}\right]
\end{aligned}
$$

The statement of the above theorem follows in view of equations (1.24), (1.25), (2.1), (2.2), (1.19), (1.20) and (2.6).

Theorem (2.2). If a hyperbolically Kaehlerian space satisfies any two of the following properties:

(i) the space is hyperbolically Kaehlerian Ricci-bi-recurrent ,

(ii) the space is hyperbolically Kaehlerian space with bi-recurrent Weyl-Conformal curvature tensor,

(iii) the space is hyperbolically Kaehlerian space with bi-recurrent Weyl-Concircular curvature tensor , then it must also satisfy the third.

Proof. A Hyperbolically Kaehlerian Ricci -bi-recurrent space, a Hyperbolically Kaehlerian space with birecurrent Weyl-Conformal curvature tensor and hyperbolically Kaehlerian space with bi-recurrent WeylConcircular curvature tensor are respectively characterized by the equations (1.24), (2.3) and (2.4).

Differentiating (1.22) covariantly w.r.to $x^{a}$, again differentiate the result thus obtained covariantly w.r.to $x^{b}$, we have

$$
C_{i j k_{i} a b}^{h}=Z_{i j k_{a} a b}^{h}+\frac{1}{(n-2)}\left(L_{i k, a b} \delta_{j}^{h}-L_{j k_{a} a b} \delta_{i}^{h}+g_{i k} L_{j, a b}^{h}-g_{j k} L_{i, a b}^{h}\right),
$$

Multiplying (1.22) with $\lambda_{a b}$ and subtracting the result thus obtained from (2.7), we have

$$
\begin{aligned}
C_{i j k, a b}^{h}-\lambda_{a b} C_{i j k}^{h}=Z_{i j k, a b}^{h}-\lambda_{a b} Z_{i j k}^{h} & +\frac{1}{(n-2)}\left[\left(L_{i k, a b}-\lambda_{a b} L_{i k}\right) \delta_{j}^{h}-\left(L_{j k, a b}-\lambda_{a b} L_{j k}\right) \delta_{i}^{h}\right. \\
& \left.+\left(L_{j, a b}^{h}-\lambda_{a b} L_{j}^{h}\right) g_{i k}-\left(L_{i, a b}^{h}-\lambda_{a b} L_{i}^{h}\right) g_{j k}\right],
\end{aligned}
$$

The statement of the above theorem follows in view of (1.19), (1.20) (1.24), (2.3), (2.4) and (2.8).

Theorem (2.3). Every hyperbolically Kaehlerian bi-recurrent space is a hyperbolically Kaehlerian space with Tachibana H-Concircular bi-recurrent space.

Proof. Differentiating (1.16) covariantly w.r.to $x^{\alpha}$, again differentiate the result thus obtained covariantly w.r.t. $x^{b}$, we have

$$
T_{i j k_{a b}}^{h}=R_{i j k_{a} a b}^{h}+\frac{R_{a b b}}{n(n+2)}\left(g_{i k} \delta_{j}^{h}-g_{j k} \delta_{i}^{h}+F_{i k} F_{j}^{h}-F_{j k} F_{i}^{h}+2 F_{i j} F_{k}^{h}\right)
$$

Multiplying (1.16) by $\lambda_{a b}$ and subtracting the result thus obtained from (2.9), we have $T_{i j k_{a} a b}^{h}-\lambda_{a b} T_{i j k}^{h}=R_{i j k, a b}^{h}-\lambda_{a b} R_{i j k}^{h}+\frac{\left(R_{a b}-\lambda_{a b}\right)}{n(n+2)}\left(g_{i k} \delta_{j}^{h}-g_{j k} \delta_{i}^{h}+F_{i k} F_{j}^{h}-F_{j k} F_{i}^{h}+2 F_{i j} F_{k}^{h}\right)$,

(2.10)

Now, let the space be hyperbolically Kaehlerian bi-recurrent, then equations (1.23), (1.24) and (1.25) are satisfied.

Making use of equations (1.23) and (1.25) in (2.10), we have

$$
T_{i j k_{i} a b}^{\text {h }}-\lambda_{a b} T_{i j k}^{h}=0 \text {, }
$$

which shows that the space is hyperbolically Kaehlerian space with Tachibana H-Concircular bi-recurrent space. 


\section{Hyperbolically Kaehlerian Spaces With Bi-Symmetric Properties}

Definition (3.1). A hyperbolically Kaehlerian space is said to be bi-symmetric if it satisfies the relation $R_{\mathrm{ijk}, \mathrm{ab}}^{\mathrm{h}}=0$, or, equivalently $\mathrm{R}_{\mathrm{ijk} \mathrm{kb}}=0$,

Obviously, a hyperbolically Kaehlerian bi-symmetric space is said to be hyperbolically Kaehlerian Ricci-bisymmetric space if

$R_{i j a b}=0$.

Multiplying the above equation by $g^{i j}$, we get

$R_{a b}=0$.

Definition (3.2). A hyperbolically Kaehlerian space satisfying the relation

$$
P_{i j k a b}^{h}=0 \text {, or equivalently } P_{i j k l_{\mu} a b}=0 \text {, }
$$

is called a hyperbolically Kaehlerian projective bi-symmetric space.

Definition (3.3). A hyperbolically Kaehlerian space satisfying the relation

$$
T_{i j k, a b}^{h}=0 \text {, or, equivalently } T_{i j k l_{j} a b}=0 \text {, }
$$

will be called hyperbolically Kaehlerian space with Tachibana H-Concircular bi-symmetric space.

Definition (3.4). A hyperbolically Kaehlerian space satisfying the relation

$$
C_{i j k a b}^{h}=0 \text {, or, equivalently } C_{i j k l a b}=0 \text {, }
$$

will be called hyperbolically Kaehlerian space with bi-symmetric Weyl-Conformal curvature tensor.

Definition (3.5). A hyperbolically Kaehlerian space satisfying the relation

$$
Z_{\mathrm{ijk} a b}^{\mathrm{h}}=0 \text {, or, equivalently } \mathrm{Z}_{\mathrm{ijk} \mathrm{k} \mathrm{ab}}=0 \text {, }
$$

is called hyperbolically Kaehlerian space with bi-symmetric Weyl-Concircular curvature tensor. Now, we have the following :

Theorem (3.1). If a hyperbolically Kaehlerian space satisfies any two of the following properties:

(i) the space is hyperbolically Kaehlerian Ricci-bi-symmetric,

(ii) the space is hyperbolically Kaehlerian projective bi-symmetric ,

(iii) the space is hyperbolically Kaehlerian Tachibana H-Concircular bi-symmetric, then it must also satisfy the third.

Proof. A hyperbolically Kaehlerian Ricci-bi-symmetric space, a hyperbolically Kaehlerian Projective bisymmetric space and hyperbolically Kaehlerian space with Tachibana H-Concircular bi-symmetric space are respectively characterized by (3.2), (3.4) and (3.5).

The statement of the above theorem follows in view of (2.5), (3.2), (3.4) and (3.5).

Theorem (3.2) . If a hyperbolically Kaehlerian space satisfies any two of the following properties:

(i) the space is hyperbolically Kaehlerian Ricci-bi-symmetric,

(ii) the space is hyperbolically Kaehlerian space with bi-symmetric Weyl-Conformal curvature tensor,

(iii) the space is hyperbolically Kaehlerian space with bi-symmetric Weyl-Concircular curvature tensor , then it must also satisfy the third.

Proof. A Hyperbolically Kaehlerian Ricci -bi-symmetric space, a Hyperbolically Kaehlerian space with bisymmetric Weyl - Conformal curvature tensor and hyperbolically Kaehlerian space with bi-symmetric WeylConcircular curvature tensor are respectively characterized by (3.2), (3.6) and (3.7).

The statement of the above theorem follows in view of (2.7), (3.2), (3.6) and (3.7).

Theorem (3.3). Every hyperbolically Kaehlerian bi-symmetric space is a hyperbolically Kaehlerian space with Tachibana H-Concircular bi-symmetric space.

Proof. From (2.9), it follows that in a hyperbolically Kaehlerian bi-symmetric space, the Tachibana HConcircular curvature tensor satisfies

$$
T_{i j k a b}^{h}=0,
$$

which shows that the space is hyperbolically Kaehlerian space with Tachibana H-Concircular bi-symmetric space. 


\section{References}

[1] Okumura, M.: Some remarks on space with a certain contact structures, Tohoku Math. Jour., 14, (1962) 135 - 145

[2] Tachibana, S.: On the Bochner curvature tensor, Nat. Sci. Report Ochanomizu Univ., 18(1), (1967)15-19.

[3] Singh, S.S.: On Kaehlerian spaces with recurrent Bochner curvature tensor, Acc. Naz. Dei. Lincei, Rend, Series VIII, 51, 3-4, (1971) 213-220.

[4] Singh, S.S.: On Kaehlerian recurrent and Ricci-recurrent spaces of second order, Atti della Acad-emia delle Scienze di Torino, 106, 11, (1971-72) 509-518.

[5] Yano, K.: Differential Geometry of Complex and almost complex spaces, Pergamon press (1965).

[6] Negi, D.S. \& Rawat, K.S. : Some bi-recurrence and bi-symmetric properties in a Kaehlerian space, Acta Ciencia Indica, Vol. XX M, No. 1 (1994) 95-100.

[7] Rawat, K.S. \& Silswal, G.P. : Some theorems on subspaces of subspaces of a Tachibana spaces,

[8] Acta Ciencia Indica, Vol. XXXI M, No. 4, (2005) 1009-1016.

[9] Rawat,K.S.\&Silswal,G.P.:TheoryofLie-derivativesandmotionsinTachibanaspaces,News Bull. Cal. Math. Soc. , 32 (1-3), (2009) 1520.

[10] Rawat,K.S.\&PrasadVirendra:SometheoremsonKaehlerianConharmonicbi-recurrent spaces,Acta Ciencia indica, Vol. XXXV M, No. 2, (2009) 417-421 MR 2666522.

[11] RawatK.S.\&PrasadVirendra:OnLie-derivativesofscalars,VectorsandTensors,Purea Applied Mathematica Sciences, Vol. LXX, No. $1-2,(2009) 135-140$.

[12] Rawat, K.S. \& Dobhal Girish : On Einstein-Kaehlerian s-recurrent space, International Trans. in

[13] Mathematical Sciences and Computer, Vol. 2, No. 2, (2009) 339-343.

[14] Rawat,K.S.\&MukeshKumar:OncurvaturecollineationsinaTachibanarecurrentspace, Aligarh Bull. Math. , 28 , No. 1-2 , (2009) 6369 MR 2769016.

[15] Rawat,K.S.\&UniyalNitin:StudyonKaehlerianrecurrentandsymmetricspacesofsecond order, Jour. of the Tensor Society, Vol. 4 , (2010) 69-76.

[16] Rawat, K.S. \& Prasad Virendra : On Holomorphically Projectively flat parabolically Kaehlerian

[17] spaces, Rev. Bull. Cal. Math. Society, 18 (1), (2010) 21-26.

[18] Rawat,K.S.\&DobhalGirish:ThestudyofTachibanabi-recurrentspaces,AntarcticaJour. Math. ,7(4) , (2010) 413-420.

[19] Rawat, K. S. \& Kumar Mukesh : On hyper surfaces of a Conformally flat Kaehlerian recurrent

[20] space, Pure and Applied Mathematika Sciences, Vol. LXXIII, No. 1-2, (2011) 7-13.

[21] Rawat,K.S.\&UniyalNitin:OnConformaltransformationsinanalmostKaehlerian and Kaehlerian spaces, Jour. of Progressive Science , Vol. 2 , No. 2 , (2011) 138-141. 